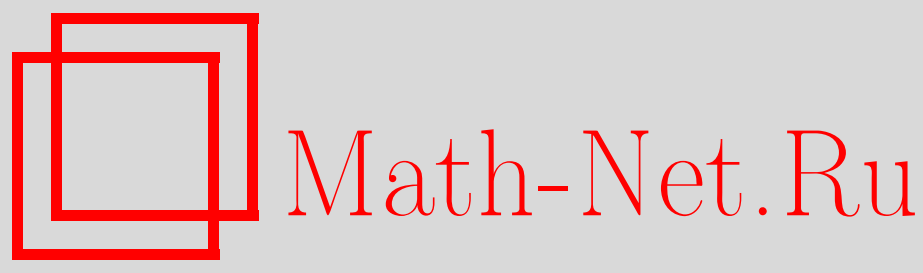

Н. А. Тюрин, Специальные лагранжевы слоения многообразия флагов $F^{3}, T M \Phi, 2011$, том 167, номер 2, 193205

DOI: https://doi.org/10.4213/tmf6633

Использование Общероссийского математического портала Math-Net.Ru подразумевает, что вы прочитали и согласны с пользовательским соглашением http://www.mathnet.ru/rus/agreement

Параметры загрузки:

IP : 54.147 .182 .235

26 апреля 2023 г., 15:28:31

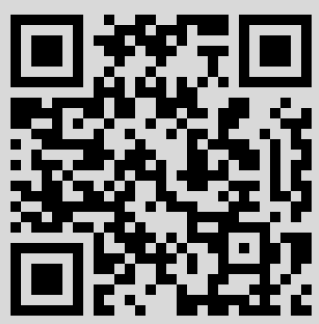




\title{
СПЕЦИАЛЬНЫЕ ЛАГРАНЖЕВЫ СЛОЕНИЯ МНОГООБРАЗИЯ ФЛАГОВ $F^{3}$
}

\begin{abstract}
Предложена конструкция слоения на лагранжевы торы полного многообразия флагов в $\mathbb{C}^{3}$. В отличие от классического слоения, получаемого из системы Гельфанда-Цейтлина, предлагаемое слоение является специальным лагранжевым.
\end{abstract}

Ключевые слова: многообразие флагов, лагранжев тор, псевдоторическая структура, специальное лагранжево слоение.

\section{1. ВВЕДЕНИЕ}

Лагранжевы слоения симплектических многообразий важны для нескольких приложений. С точки зрения классической механики, если фазовое пространство системы расслоено на лагранжевы подмногообразия, то движение в системе с гамильтонианом, векторное поле которого паралелльно слоению, описывается в терминах слоев, и это имеет место даже в том случае, когда некоторые слои являются особыми.

Один из подходов, принятых в геометрическом квантовании $(Г \mathrm{~K})$, реализуется в такой же ситуации: если данное фазовое пространство расслоено на лагранжевы подмногообразия, то выбираются так называемые бор-зоммерфельдовы слои, составляющие дискретное подмножество, и этот набор порождает соответствующее гильбертово пространство. Здесь необходимо отметить, что понятие бор-зоммерфельдова лагранжева подмногообразия остается рабочим для некоторых типов особых лагранжевых слоев. Гомологическая зеркальная симметрия (ГЗС) сегодня включает в себя рассмотрение лагранжевых слоений и выделение в них конечного подмножества слоев, удовлетворяющих некоторому условию в терминах когомологий Фукаи-Флоера. И в этом случае тоже можно попробовать произвести подсчет голоморфных дисков для некоторых особых торов, так что и этот подход к ГЗС может быть продолжен на случай, когда слоения допускают особые слои.

*Объединенный институт ядерных исследований, Дубна, Московская обл., Россия. E-mail: ntyurin@theor.jinr.ru

${ }^{\dagger}$ Государственный университет - Высшая школа экономики, Москва, Россия

${ }^{\ddagger}$ Московский государственный университет путей сообщения (МИИТ), Москва, Россия 
Идеальная модельная ситуация для наших рассуждений содержится в торической геометрии [1]. Если мы работаем с симплектическим торическим многообразием, т. е. с фазовым пространством вполне интегрируемой системы, то существует каноническое лагранжево слоение, параметризуемое выпуклым многогранником, что приводит к введению переменных действие-угол и полному решению системы. В то же время методы ГК и ГЗС, упомянутые выше, прекрасно работают в торическом случае. Проблема состоит в том, что множество торических многообразий не столь велико, и существуют другие многообразия, которые не обладают торической структурой по топологическим причинам, но должны быть вовлечены в рассмотрение.

Для компактного симплектического торического многообразия $(M, \omega)$ каноническое лагранжево слоение не является вполне регулярным - имеет место размерностная редукция: гладкие лагранжевы торы заполняют полностью не всё $M$, а только дополнение $M \backslash D$, где $D$ - “симплектический дивизор", класс гомологий которого двойствен по Пуанкаре антиканоническому классу $M$. Симплектическое подмногообразие $D$ вещественной коразмерности 2 состоит из некоторого числа неприводимых компонент $D_{i}$. Каждая из этих компонент как симплектическое многообразие расслоено на лагранжевы торы, которые являются изотропными торами в $M$. Общая картина описывается в терминах многогранника моментов $P$ : каждая его грань есть многогранник моментов для соответствующего симплектического торического многообразия вещественной размерности, равной удвоенной размерности грани. С этой точки зрения естественно называть $D$ граничным дивизором.

Расширим класс симплектических торических многообразий. В настоящей работе, как и в статьях [2], [3], мы изучаем компактные гладкие симплектические многообразия, допускающие лагранжевы слоения почти такого же типа, что и в торическом случае, но разрешаем наличие особых торов в слоениях. Мы требуем, чтобы общий слой был гладким, а типы разрешенных особенностей исчерпывались следующей моделью. Для гладкого $n$-мерного тора $T^{n}$ возьмем произвольное разложение

$$
T^{n}=T^{k} \times T^{n-k}
$$

и применим к $T^{n}$ такую процедуру: в произвольной точке $p \in T^{n-k}$ слой $T_{p}^{k}=$ $T^{k} \times\{p\}$ сжимается до $(k-1)$-мерного тора. Обозначим соответствующее преобразование через $C_{p}^{k}$ и скажем, что особый тор, разрешенный в наших слоениях, должен иметь вид $C_{p_{1}}^{k_{1}} \ldots C_{p_{l}}^{k_{l}}\left(T^{n}\right)$. Заметим, что этот тип особенностей хорошо известен в классической механике: такие особенности соответствуют сепаратрисам. В то время как гладкий тор в слоении несет на себе траектории типа петель, особый тор несет на себе также прямые и неподвижные точки как решения.

В настоящей статье мы строим лагранжевы слоения описанного выше типа на комплексном многообразии флагов $F^{3}$, являющемся полным флаговым многообразием для $\mathbb{C}^{3}$. Ниже описывается лагранжево слоение многообразия $F^{3}$ с граничным дивизором $D$, составленным из четырех поверхностей дель Пеццо $\mathbb{C P}_{1}^{2}$, и с подсемейством особых торов вещественной размерности 1. Общий слой этого слоения есть гладкий лагранжев тор, а особый слой получается применением преобразования $C_{p}^{2}$ к $T^{3}$. Это не единственное слоение, которое дает наша конструкция, но мы выделяем именно его и называем минимальным, так как оно обладает минимальным 
подсемейством особых торов. Наша конструкция основана на том, что $F^{3}$ обладает псевдоторической структурой, представляющей собой некоторое обобщение понятия торической структуры, предложенное в работах [2] и [3]. Всякое псевдоторическое многообразие обладает множеством лагранжевых слоений описанного типа.

На $F^{n}$, полном многообразии флагов в $\mathbb{C}^{n}$, имеется известная система Гельфанда-Цейтлина, вполне интегрируемая, но не являющаяся эффективной, поскольку $F^{n}$ - не торическое многообразие. Образ многообразия $F^{n}$ при отображении “действия" есть многогранник Гельфанда-Цейтлина. Отличие от торического случая выражается в нарушении размерностной редукции: некоторые грани многогранника Гельфанда-Цейтлина не являются образами комплексных подмножеств в $F^{n}$ и даже образами некоторых подмножеств правильной размерности в $F^{n}$ (т. е. размерности, равной удвоенной размерности грани). Например, существуют вершины, прообразы которых не являются точками в $F^{n}$ (существует вершина максимального вырождения, прообраз которой - лагранжева $n$-мерная сфера). Таким образом, наша конструкция, в отличие от системы Гельфанда-Цейтлина, предлагает альтернативный путь построения лагранжева слоения на $F^{3}$ - мы сохраняем аргументы размерностной редукции, как и в торическом случае, но теряем гладкость слоев. В самом деле, граничный “дивизор" для системы Гельфанда-Цейтлина на многообразии флагов $F^{3}$ состоит из шести компонент, двух комплексных и четырех некомплексных, и имеется единственная четырехвалентная вершина (лежащая на пересечении этих четырех компонент) в многограннике Гельфанда-Цейтлина, прообраз которой - лагранжева сфера в $F^{3}$. Граничный дивизор нашего минимального лагранжева слоения состоит из четырех комплексных компонент.

Чем интересны минимальные лагранжевы слоения на $F^{3}$ ? Во-первых, они дают новые специальные решения классической механической задачи на $F^{3}$. Например, можно заметить, что некоторая комбинация трех функций $\lambda_{j}^{i}$ из системы Гельфанда-Цейтлина дает две функции $f_{1}, f_{2}$ из нашей конструкции. Это означает, что если взять на $F^{3}$ гамильтониан $H$ вида

$$
H=\alpha f_{1}+\beta f_{2}, \quad \alpha, \beta \in \mathbb{R},
$$

то траектории движения, генерируемого $H$, могут быть найдены двумя способами: путем рассмотрения слоев лагранжева слоения Гельфанда-Цейтлина, по которым осуществляется движение, или путем рассмотрения минимального лагранжева слоения и изучения движения на нем. Ответ, безусловно, должен быть один и тот же, однако в некоторых вопросах может оказаться более удобным работать с минимальным лагранжевым слоением, например при отыскании минимальных орбит.

Во-вторых, можно применять стандартные методы ГК к многообразию флагов $F^{3}$, снабженному минимальным лагранжевым слоением. Для особого тора, включающегося в это слоение, понятие бор-зоммерфельдова лагранжева цикла остается корректным: сжатый тор обладает стягиваемым в точку подмножеством "виртуальных" периодов, соответствующих образующим в фундаментальной группе, и эти виртуальные периоды должны быть тривиальными относительно любой целочисленной симплектической формы. Следовательно, можно определить множество бор-зоммерфельдовых слоев (относительно антиканонического класса, так как $F^{3}$ 
является многообразием Фано). Можно показать, что это множество является дискретным и конечным, таким образом, гильбертово пространство натягивается, как обычно, на это конечное множество. Преимущество минимального лагранжева слоения состоит в явной связи между лагранжевым и кэлеровым квантованиями многообразия $F^{3}$ : как и в классическом торическом случае имеется версия соответствия Данилова-Хованского между голоморфными сечениями антиканонического класса и лагранжевыми циклами, удовлетворяющими условию Бора-Зоммерфельда относительно антиканонического класса. Необходимо напомнить, что для этих задач существует классический результат [4], решающий проблему ГК в терминах теории представлений.

В-третьих, при изучении специалъных лагранжевых слоений на многообразиях Фано, введенных недавно в работе [5], если мы исследуем в этой связи многообразие флагов $F^{3}$ (или, в более общем случае, многообразие $F^{n}$ ), то слоение Гельфанда-Цейтлина не может обладать свойством специальности и, более того, не может привести к слоению искомого вида. В то же время минимальное лагранжево слоение на $F^{3}$, построенное ниже, является специальным лагранжевым, и этот факт наиболее интересен для нас. Последние рассуждения носят достаточно специальный характер, но все же наша конструкция может в дальнейшем привести к содержательным результатам и в лагранжевой геометрии многообразий Фано.

\section{2. ПСЕВДОТОРИЧЕСКИЕ СТРУКТУРЫ НА МНОГООБРАЗИИ ФЛАГОВ $F^{3}$}

Мы строим большое семейство лагранжевых слоений на $F^{3}$, используя понятие псевдоторической структуры, введенное в работах [2], [3]. Справедливо следующее

ПреДЛОЖЕНИЕ 1. Многообразие флагов $F^{3}$ является псевдоторическим.

Напомним, псевдоторическая структура на симплектическом многообразии $\left(M, \omega_{M}\right)$ вещественной размерности $2 n$ задается набором данных $\left(f_{1}, \ldots, f_{k}, B, \psi,\left(Y, \omega_{Y}\right)\right)$, где $f_{i}$ - гладкие морсовские функции в инволюции, $\psi$ определяет семейство симплектических торических многообразий с базисным множеством $B \subset M$, параметризуемое симплектическим торическим многообразием $\left(Y, \omega_{Y}\right)$ вещественной размерности $2(n-k)$ так, что

$$
\psi: M-B \rightarrow Y
$$

есть отображение с общим гладким симплектическим слоем, причем выполнены следующие условия согласования:

1) для каждого $p \in Y$ соответствующий элемент семейства

$$
\overline{\psi^{-1}(p)}=\psi^{-1}(p) \cup B
$$

сохраняется при гамильтоновом действии каждой из $f_{i}$;

2) для любой гладкой функции $h \in C^{\infty}(Y, \mathbb{R})$ имеет место тождество

$$
\nabla_{\psi} X_{h} \wedge X_{\psi^{*} h} \equiv 0
$$

на $M-(B \cup \operatorname{Sing})$, где Sing - множество особенностей особых слоев отображения $\psi$, $\nabla_{\psi}$ - симплектическая связность, порождаемая $\psi$, а $X_{h}$ и $X_{\psi^{*} h}$ - гамильтоновы векторные поля, определяемые $\omega_{Y}$ и $\omega_{M}$ соответственно. 
Иными словами, если имеется торическое действие $T^{k}$ на $M$, то мы рассматриваем случай, когда листы этого действия, т. е. семейство (для общего элемента гладких) симплектических торических многообразий с одними и теми же отображениями моментов, параметризуется симплектическим торическим многообразием. Заметим, что второе условие согласованности выполнено автоматически, если и $\left(M, \omega_{M}\right)$, и $\left(Y, \omega_{Y}\right)$ являются кэлеровыми многообразиями, $\psi$ - комплексное отображение, а комплексная размерность $Y$ равна единице.

Как было доказано в работе [3], если зафиксировать псевдоторическую структуру $\left(f_{1}, \ldots, f_{k}, B, \psi,\left(Y, \omega_{Y}\right)\right)$ на симплектическом многообразии $\left(M, \omega_{M}\right)$, то выбор произвольного набора отображений моментов $\left(h_{1}, \ldots, h_{n-k}\right)$ на $Y$ задает лагранжево слоение на $M$.

Теперь мы готовы к доказательству предложения 1. Рассмотрим прямое произведение $\mathbb{C P}_{x}^{2} \times \mathbb{C P}_{y}^{2}$, зафиксируем однородные координаты $\left[x_{0}: x_{1}: x_{2}\right]$ и $\left[y_{0}: y_{1}: y_{2}\right]$ на этих проективных плоскостях и реализуем $F^{3}$ как гиперповерхность в $\mathbb{C P}_{x}^{2} \times \mathbb{C P}_{y}^{2}$, задаваемую уравнением

$$
\sum_{i=0}^{2} x_{i} y_{i}=0
$$

Рассмотрим отображение

$$
\psi: \mathbb{C P}_{x}^{2} \times \mathbb{C P}_{x}^{2}-B \rightarrow \mathbb{C P}_{w}^{2},
$$

определяемое соотношениями

$$
w_{i}=x_{i} y_{i}, \quad i=0,1,2,
$$

где $\left[w_{0}: w_{1}: w_{2}\right]$ - однородные координаты на $\mathbb{C P}_{w}^{2}$. Так как при фиксации координат одновременно фиксируется соответствующая метрика Фубини-Штуди, все проективные плоскости снабжаются соответствующими симплектическими формами. Базисное множество $B$ состоит из шести прямых, определяемых условиями

$$
x_{i}=x_{j}=y_{k}=0 \quad \text { или } \quad x_{i}=y_{j}=y_{k}=0,
$$

где $i, j, k$ - различные числа из множества $\{0,1,2\}$.

Что является слоями отображения $\psi$ ? Нетрудно видеть, что для произвольной точки $p \in \mathbb{C P}_{w}^{2}$ с координатами $\left[w_{0}: w_{1}: w_{2}\right]$ такими, что $w_{i} \neq 0$ для всех $i=0,1,2$, компактифицированный слой

$$
\overline{\psi^{-1}(p)}=\psi^{-1}(p) \cup B
$$

есть поверхность дель Пеццо, изоморфная $\mathbb{C P}_{3}^{2}$. Базисное множество $B$ - комплексный шестиугольник в $\mathbb{C P}_{3}^{2}$. Такой слой является общим.

Если взять теперь точку $p \in \mathbb{C P}_{w}^{2}$ с координатами $\left[w_{0}: w_{1}: w_{2}\right]$ так, что в точности одна из $w_{i}$ равна нулю, то соответствующим компактифицированным слоем $\overline{\psi^{-1}(p)}$ будет объединение двух поверхностей дель Пеццо вида $\mathbb{C P}_{1}^{2}$, пересекающихся по “диагонали" шестиугольника $B$. Каждая из этих плоскостей имеет базисный четырехугольник, граница которого составлена из трех сторон шестиугольника $B$ 
и прямой пересечения поверхностей. Таким образом, этот компактифицированный слой является особым, и его особое множество есть в точности “диагональная" прямая.

Для точки $p \in \mathbb{C P}_{w}^{2}$ с координатами $\left[w_{0}: w_{1}: w_{2}\right]$, из которых только одна $w_{i}$ ненулевая, компактифицированный слой снова будет особым. Он состоит из двух $\mathbb{C P}^{2}$ и двух квадрик. Шестиугольник $B$ делится при этом на два треугольника и два четырехугольника парой пересекающихся диагоналей; треугольники лежат в плоскостях, а четырехугольники - в квадриках.

Топологически каждый слой является торическим, и наша конструкция снабжает каждый слой дивизором из антиканонической системы, который может рассматриваться как симплекс вырождения соответствующего набора отображений моментов (или граничный дивизор). Покажем, что $\mathbb{C P}_{x}^{2} \times \mathbb{C P}_{y}^{2}$ несет на себе пару отображений моментов, гамильтоново действие которых сохраняет каждый слой, т. е. их ограничения на каждый слой дают вполне интегрируемую систему.

Для плоскости $\mathbb{C P}_{x}^{2}$ в прямом произведении рассмотрим вещественную функцию вида

$$
f(x)=\frac{\sum_{i=0}^{2} \lambda_{i}^{x}\left|x_{i}\right|^{2}}{\sum_{i=0}^{2}\left|x_{i}\right|^{2}},
$$

где $\lambda_{i}^{x}$ - попарно различные вещественные числа. Для $\mathbb{C P}_{y}^{2}$ функция $f(y)$ получается подстановкой $y$ вместо $x$ в той же формуле. Тогда $f(x), f(y)$ - вещественные морсовские функции на соответствующих $\mathbb{C P}^{2}$, гамильтоновы действия которых сохраняют кэлеровы структуры. Если обозначить две естественные проекции как

$$
q_{*}: \mathbb{C P}_{x}^{2} \times \mathbb{C P}_{y}^{2} \rightarrow \mathbb{C P}_{*}^{2}, \quad *=x, y,
$$

то функция $F=q_{x}^{*} f(x)+q_{y}^{*} f(y)$ (где звездочка теперь обозначает подъем) будет вещественной морсовской функцией на прямом произведении, гамильтоново векторное поле которой сохраняет кэлерову структуру на $\mathbb{C P}_{x}^{2} \times \mathbb{C P}_{y}^{2}$. Нетрудно видеть, что если

$$
\lambda_{0}^{x}+\lambda_{0}^{y}=\lambda_{1}^{x}+\lambda_{1}^{y}=\lambda_{2}^{x}+\lambda_{2}^{y},
$$

то гамильтоново векторное поле такой функции $F$ должно сохранять слои отображения $\psi$. Так как на $\mathbb{C P}^{2}$ мы имеем в точности две алгебраически независимые почти всюду вещественные морсовские функции такого вида, существуют в точности две алгебраически независимые почти всюду морсовские функции $F_{1}, F_{2}$, сохраняющие слои. Симплекс вырождения

$$
\Delta\left(F_{1}, F_{2}\right)=\left\{X_{F_{1}} \wedge X_{F_{2}}=0\right\} \subset \mathbb{C P}_{x}^{2} \times \mathbb{C P}_{y}^{2}
$$

состоит из шестиугольника $B$ и трех его "диагоналей". Таким образом, согласно общей теории (см. статью [3]) симплекс вырождения есть в точности объединение $B$ и Sing.

Мы утверждаем, что построенные данные $\left(F_{1}, F_{2}, B, \psi, \mathbb{C P}^{2}\right)$ задают некоторую псевдоторическую структуру на прямом произведении $\mathbb{C P}_{x}^{2} \times \mathbb{C P}_{y}^{2}$. Нетрудно показать, что второе условие согласованности выполняется, мы оставляем это рассуж- 
дение за рамками настоящей работы. Сейчас наша цель - показать, что $F^{3}$ также обладает псевдоторической структурой. Данная псевдоторическая структура получается ограничением данных $\left(F_{1}, F_{2}, B, \psi, \mathbb{C P}^{2}\right)$ на слои над прямой $\mathbb{C P}_{w}^{1} \subset \mathbb{C P}_{w}^{2}$, задаваемой уравнением

$$
w_{0}+w_{1}+w_{2}=0
$$

Поскольку гамильтоновы векторные поля функций $F_{1}, F_{2}$ касательны $F^{3}$, а образом $F^{3}$ при отображении $\psi$ будет в точности прямая $\mathbb{C P}_{w}^{1}$, мы имеем набор данных $\left(f_{1}, f_{2}, B, \psi_{1}, \mathbb{C P}^{1}\right)$, где $f_{i}=\left.F_{i}\right|_{F^{3}}, B=B$, так как $B$ лежит в $F^{3}, \psi_{1}=\left.\psi\right|_{F^{3}-B}$. Нетрудно видеть, что набор данных $\left(f_{1}, f_{2}, B, \psi_{1}, \mathbb{C P}_{w}^{1}\right)$ задает псевдоторическую структуру на $F^{3}$. Предложение доказано.

Прямая $\mathbb{C P}_{w}^{1}=\left\{w_{0}+w_{1}+w_{2}=0\right\} \subset \mathbb{C P}^{2}$ пересекает три базисные прямые $l_{i}=\left\{w_{i}=0\right\}, i=0,1,2$, в трех точках, и именно эти точки подлежат особым слоям отображения $\psi$. Тип особых слоев уже был описан выше: каждый из них есть пара $\mathbb{C P}_{1}^{2}$ (поверхностей дель Пеццо степени 8) с особым множеством, представляемым "диагональю".

Теперь мы готовы описать минимальное лагранжево слоение на $F^{3}$, упомянутое во введении. Зафиксируем гладкую морсовскую функцию на $\mathbb{C P}^{1}$, имеющую две критические точки $[0: 1:-1]$ и $[1: 0:-1]$ - максимум и минимум. Тогда граничный дивизор $D \subset F^{3}$ соответствующего слоения состоит из двух особых слоев, а каждый из них, в свою очередь, состоит из двух поверхностей дель Пеццо $\mathbb{C P}_{1}^{2}$. Таким образом, это дает четыре поверхности дель Пеццо, каждая из которых является торической с интегралами, получаемыми ограничением $F_{1}, F_{2}$ на каждую компоненту. Эти интегралы индуцируют каноническое торическое слоение на каждой компоненте. Это показывает, что $D$ обладает слоением на гладкие торы, как обычно в торической геометрии. Рассмотрим теперь, что происходит на $F^{3}-D$. Каждый слой $\psi^{-1}(p)$ расслаивается на гладкие двумерные торы путем ограничения функций $f_{1}$ и $f_{2}$. Эти торы нумеруются парой регулярных значений функций $f_{1}$ и $f_{2}$. Возьмем гладкую линию уровня - петлю $\gamma-$ функции $h$ на $\mathbb{C P}^{1}$, не проходящую через точку $[1:-1: 0]$. Зафиксируем пару регулярных значений для $f_{1}, f_{2}$ и соберем соответствующие им торы вдоль петли $\gamma$ : возьмем $\bigcup_{p \in \gamma} T_{p}^{c_{1}, c_{2}}$, где $T_{p}^{c_{1}, c_{2}}-$ тор из слоя над $p$ со значениями $c_{1}, c_{2}$. Тогда такое объединение даст гладкий трехмерный тор, являющийся лагранжевым в $F^{3}$. Это случай общего слоя.

Когда возникают особые торы? Если $\gamma$ проходит через особую точку $[1:-1: 0]$, то это еще не означает автоматически, что соответствующий тор получится особым. В самом деле, для внутренних точек шестиугольника в $\mathbb{R}^{2}$, образованных парой значений функций $f_{1}, f_{2}$, тор в слое над $[1:-1: 0]$ будет двумерным или одномерным, если пара значений $\left(c_{1}, c_{2}\right)$ лежит на диагонали шестиугольника. В общем случае $\left(c_{1}, c_{2}\right)$ дает двумерный тор, поэтому, собирая торы вдоль $\gamma$, мы снова получим гладкий лагранжев тор. Особенность возникает, когда точка $\left(c_{1}, c_{2}\right)$ попадает на диагональ, поэтому этот отрезок параметризует особые торы, имеющие следующий тип: операция $C_{p}^{2}$ применена к $T^{3}$.

Представленное выше лагранжево слоение $F^{3}$ является минимальным в следующем смысле: другой выбор функции $h$ на прямой $\mathbb{C P}^{1}$, подлежащей $\psi_{1}$, даст другое 
слоение с бо́льшим множеством особых торов (за исключением случая, когда $h$ удовлетворяет тем же свойствам, т. е. ее минимум и максимум совпадают с какой-то другой парой из трех выделенных точек).

\section{3. УСЛОВИЕ СПЕЦИАЛЬНОСТИ}

Напомним, что изначально условие специальности для лагранжевых торов было введено для многообразий Калаби-Яу как условие, “двойственное" условию голоморфности в рамках проблемы ГЗС. В работе [5] это условие обобщается на случай некоторых многообразий Фано: если многообразие Фано $X$ допускает эффективный дивизор в антиканонической системе, $D \subset\left|-K_{X}\right|$, то существует старшая голоморфная форма $\theta_{D}$ на дополнении $X-D$; эта форма имеет полюс в $D$ и не обращается в нуль на $X-D$. Заметим, что эта форма однозначно определяется дивизором $D$ с точностью до $\mathbb{C}^{*}$.

Так как по определению многообразие Фано $X$ может быть вложено в проективное пространство некоторой степенью своей антиканонической системы, можно приписать многообразию $X$ соответствующую симплектическую форму, которая получается как ограничение стандартной симплектической формы на проективном пространстве на образ при вложении $X$. Таким образом, произвольное многообразие Фано несет некоторую симплектическую структуру с симплектической формой, класс когомологий которой пропорционален антиканоническому классу. Поэтому любое многообразие Фано можно рассматривать как монотонное симплектическое многообразие. Такая симплектическая структура на $X$ может быть названа канонической (или антиканонической, если угодно).

Лагранжев тор $S \subset X$ называется специальным лагранжевым тором в $(X, D)$, если мнимая часть $\left.\operatorname{Im} e^{i s} \theta_{D}\right|_{S}$ ограничения $\left.\theta_{D}\right|_{S}$ тождественно обращается в нуль после некоторого поворота $e^{i s} \in U(1)$. Лагранжево слоение $X-D$ называется сneииалъным, если такое условие выполнено для любого слоя с одним и тем же $e^{i s}$.

Простейший пример специального лагранжева слоения можно найти в торической геометрии. В самом деле, каноническое лагранжево слоение торического многообразия $X$ получается в результате размерностного вырождения на дивизоре $D_{0}$ из антиканонической системы $\left|-K_{X}\right|$. В этом случае соответствующая старшая голоморфная форма $\theta_{D_{0}}$ имеет замечательно простой вид в координатах действие-угол, откуда следует свойство специальности.

Однако стандартное слоение не является подходящим для разного рода задач, например неудобна для дальнейших построений приводимость граничного дивизора $D_{0}$. В работе [5] была сформулирована проблема построения специального лагранжева слоения для произвольного гладкого элемента антиканонической системы на $\mathbb{C P}^{2}$. В примере нестандартного специального лагранжева слоения, предложенном в [5], граничный дивизор приводи́м, поскольку составлен из прямой и коники, однако этот случай является более общим, нежели торический. В то же время эта проблема может быть исследована для любого многообразия Фано с эффективным дивизором в антиканонической системе. Например, мы утверждаем, что многообразие флагов $F^{3}$ обладает специальным лагранжевым слоением, а именно минимальное лагранжево слоение, построенное выше, удовлетворяет следующему условию.

ПрЕДЛОЖЕНИЕ 2. Минималъное лагранжево слоение на $F^{3}$ является специалъным лагранжевым. 
В самом деле, граничный дивизор минимального слоения состоит из двух компактифицированных слоев отображения $\psi$. Антиканонический класс многообразия $F^{3}$ как расслоение представляется произведением $q_{x}^{*} \mathcal{O}_{\mathbb{C P}_{x}^{2}}(2) \otimes q_{y}^{*} \mathcal{O}_{\mathbb{C P}_{y}^{2}}(2)$ согласно формуле присоединения, в то же время компактифицированные слои отображения $\psi$ являются нулями сечений расслоения $q_{x}^{*} \mathcal{O}_{\mathbb{C P}_{x}^{2}}(1) \otimes q_{y}^{*} \mathcal{O}_{\mathbb{C P}_{y}^{2}}(1)$. Таким образом, граничный дивизор минимального слоения лежит в антиканонической системе, и гомологическое условие удовлетворено.

Возьмем теперь граничный дивизор $D \subset F^{3}$ минимального лагранжева слоения и рассмотрим соответствующую старшую голоморфную форму $\theta_{D}$ на $F^{3}-D$. Мы утверждаем, что в $F^{3}-D$ существуют три вещественных векторных поля, два глобально и одно локально, которые сохраняют голоморфную форму $\theta_{D}$. Это означает, что производная Ли формы $\theta_{D}$ относительно любого из трех этих векторных полей равна нулю. Поскольку форма $\theta_{D}$ замкнута, а векторные поля коммутируют между собой, при подстановке этой тройки векторных полей в $\theta_{D}$ получается постоянная функция. Эту тройку векторных полей образуют гамильтоновы векторные поля $X_{f_{1}}, X_{f_{2}}$ (определенные глобально на всем $F^{3}$ ) и поднятое векторное поле $\nabla_{\psi} X_{h}$, определенное вне объединения особенностей слоев отображения $\psi$. В самом деле, каждое поле $X_{f_{i}}$ порождает гамильтонову изотопию многообразия $F^{3}$, которая оставляет неподвижным граничный дивизор $D$, что влечет тривиальность производной Ли $\mathcal{L}_{X_{f_{i}}} \theta_{D}$. С другой стороны, гамильтоново векторное поле $X_{h}$ на базе псевдоторической структуры порождает некоторое однопараметрическое семейство симплектоморфизмов многообразия $F^{3}$, которые переводят слои отображения $\psi$ в слои и оставляют на месте граничный дивизор. Это означает что производная Ли $\mathcal{L}_{\nabla_{\psi}\left(X_{h}\right)} \theta_{D}$ тожественно равна нулю (но на этот раз она определена только вне особенностей отображения $\psi$ ).

Рассмотрим теперь произвольный тор $S \subset F^{3}$ из нашего минимального лагранжева слоения. Обозначим через $S_{0} \subset S$ "гладкую" часть, состоящую из гладких точек (в общем случае $S_{0}=S$ ). В произвольной точке $p \in S_{0}$ касательное пространство $T_{p} S$ натянуто на $X_{f_{1}}, X_{f_{2}}, \nabla_{\psi} X_{h}$. В то же время, поскольку $\theta_{D}\left(X_{f_{1}}, X_{f_{2}}, \nabla_{\psi} X_{h}\right)$ является постоянной комплексной функцией на $S_{0}$, а детерминантное пространство $\operatorname{det} T_{p} S$ является вещественным и одномерным, ограничение $\theta_{D}$ на $S_{0}$ должно быть пропорционально произвольной вещественной старшей форме, не обращающейся в нуль, с коэффициентом пропорциональности $f e^{i \phi}$, где $f$ - вещественная функция, $\phi$ - константа. Ключевым является тот факт, что эта константа (т.е. аргумент) одна и та же для всех торов $S \subset F^{3}$ минимального слоения. Это завершает доказательство предложения 2. Здесь мы неявно использовали тот важный факт, что функция $h$ является символом на базе $\mathbb{C P}_{w}^{1}$, т. е. ее гамильтоново векторное поле сохраняет комплексную структуру. Поэтому утверждение предложения 2 остается справедливым для любых лагранжевых слоений, индуцируемых символами на базе $\mathbb{C P}_{w}^{1}$.

\section{4. ТОРИЧЕСКОЕ ВЫРОЖДЕНИЕ МНОГООБРАЗИЯ ФЛАГОВ $F^{3}$}

Мы могли бы описать псевдоторическую структуру $\left(f_{1}, f_{2}, B, \psi_{1}, \mathbb{C P}^{1}\right)$ на многообразии флагов $F^{3}$ без всяких ссылок на его представление гиперповерхностью в прямом произведении $\mathbb{C P}_{x}^{2} \times \mathbb{C P}_{y}^{2}$, однако вычисления, приведенные выше, оказываются полезными при изучении торического вырождения многообразия $F^{3}$ и связей между его каноническим лагранжевым слоением и минимальным лагранжевым слоением $F^{3}$, построенным выше. 
Торическое вырождение многообразия $F^{3}$ - это деформация многообразия флагов в некоторое особое торическое многообразие. В координатах $\left\{x_{i}, y_{i}\right\}$ она задается следующим семейством:

$$
F_{t}=\left\{t x_{0} y_{0}+x_{1} y_{1}+x_{2} y_{2}=0\right\} \subset \mathbb{C P}_{x}^{2} \times \mathbb{C P}_{y}^{2}
$$

где $t$ - комплексный параметр. Если $t \neq 0$, то многообразие $F_{t}$ изоморфно $F^{3}$, в то время как $t=0$ влечет, что $F_{0}$ - особое торическое многообразие. Как торическое многообразие, $F_{0}$ обладает каноническим лагранжевым слоением. Наша цель в этом разделе - связать слои лагранжева слоения $F^{3}$, построенного в предыдущем разделе, со слоями этого канонического лагранжева слоения.

Первое наблюдение, ведущее в этом направлении, следующее.

ПрЕДЛОЖЕНИЕ 3. Особое торическое многообразие $F_{0}$ обладает nсевдоторической структурой, и каноническое торическое слоение может быть реализовано путем выбора подходящей функиии $h$ на базе $\mathbb{C P}^{1}$.

Рассмотрим снова $\mathbb{C P}_{x}^{2} \times \mathbb{C P}_{y}^{2}$ и псевдоторическую структуру $\left(F_{1}, F_{2}, B, \psi, \mathbb{C P}_{w}^{2}\right)$, представленную выше. Возьмем теперь прямую

$$
\mathbb{C P}_{0}^{1}=\left\{w_{1}+w_{2}=0\right\} \subset \mathbb{C P}_{w}^{2} .
$$

Очевидно, что гамильтоновы действия функций $F_{1}$ и $F_{2}$ сохраняют $F_{0}$, поэтому ограничения $F_{i}$ на $F_{0}$ дают набор коммутирующих функций; $B$ является базой семейства, ограничение отображения $\psi$ на $F_{0}$ дает отображение в $\mathbb{C P}_{0}^{1}$, которое мы обозначим через $\psi_{0}$, и всё вместе дает псевдоторическую структуру на $F_{0}$.

Особые слои отображения $\psi_{0}$ суть слои над точками $[1: 0: 0]$ и $[0: 1:-1]$ в $\mathbb{C P}_{0}^{1}$. Теперь мы можем восстановить каноническое лагранжево слоение на $F_{0}$.

ПреДЛОЖЕНИЕ 4. Функиия $h$ на $\mathbb{C P}_{0}^{1}$, имеющая в точности две критические точки, совпадающие с $[1: 0: 0]$ u $[0: 1:-1]$, порождает каноническое торическое слоение на $F_{0}$.

Это может быть показано в два приема. Во-первых, очевидно, что стандартное торическое слоение на $F_{0}$ может быть построено из некоторого канонического торического слоения на объемлющем многообразии $\mathbb{C P}_{x}^{2} \times \mathbb{C P}_{y}^{2}$, инвариантном относительно гамильтонова действия функций $F_{1}$ и $F_{2}$. Во-вторых, можно показать, что каноническое торическое слоение на прямом произведении $\mathbb{C P}_{x}^{2} \times \mathbb{C P}_{y}^{2}$ порождается некоторым выбором отображений моментов $H_{1}, H_{2}$ на $\mathbb{C P}_{w}^{2}$ в присутствии псевдоторической структуры $\left(F_{1}, F_{2}, B_{0}, \psi, \mathbb{C P}_{w}^{2}\right)$. Если эти функции $H_{1}, H_{2}$ выбраны диагональными в координатах $\left[w_{0}: w_{1}: w_{2}\right]$, более того, одна из них, скажем $H_{1}$, постоянна на прямой $l_{0}=\left\{w_{0}=0\right\}$, то гамильтоново векторное поле $H_{1}$ должно быть параллельно прямой $\mathbb{C P}_{0}^{1}$, и ограничение $H_{1}$ на $\mathbb{C P}_{0}^{1}$ будет функцией $h$.

Единственный существенный момент в этом рассуждении состоит в необходимости показать, что диагональные в координатах $\left[w_{0}: w_{1}: w_{2}\right]$ функции $H_{1}$ и $H_{2}$ порождают каноническое торическое слоение на $\mathbb{C P}_{x}^{2} \times \mathbb{C P}_{y}^{2}$, поэтому мы проверим только этот факт. Для того чтобы это увидеть, заметим, что для такого диагонального выбора функций $H_{1}, H_{2}$ особые слои отображения $\psi$ будут лежать в точности 
над симплексом вырождения $\Delta\left(H_{1}, H_{2}\right)$ функций $H_{1}, H_{2}$, т. е. над объединением прямых $l_{i}=\left\{w_{i}=0\right\} \subset \mathbb{C P}_{w}^{2}$. Это означает, что граничный дивизор $D$ соответствующего слоения на $\mathbb{C P}_{x}^{2} \times \mathbb{C P}_{y}^{2}$ составлен из компонент

$$
\overline{\psi^{-1}\left(l_{i}\right)}, \quad i=0,1,2,
$$

а это в точности шесть неприводимых компонент вида $q_{x}^{*}\left(l_{i}^{x}\right), q_{y}^{*} l_{i}^{y}, i=0,1,2$, где $l_{i}^{*} \subset \mathbb{C P}_{*}^{2}-$ проективная прямая,

$$
l_{i}^{x}=\left\{x_{i}=0\right\}, \quad l_{i}^{y}=\left\{y_{i}=0\right\}, \quad i=0,1,2 .
$$

Таким образом, граничный дивизор слоения, порождаемого функциями $H_{1}, H_{2}$, совпадает с граничным дивизором канонического торического слоения. Поскольку для диагональных функций $H_{1}, H_{2}$ соответствующее слоение имеет только гладкие слои, такое слоение должно быть изоморфно стандартному слоению (получаемому как прямое произведение пары клиффордовых слоений на плоскостях $\mathbb{C P}_{x}^{2}, \mathbb{C P}_{y}^{2}$ ).

Как можно сравнить лагранжевы слоения на $F_{0}$ и $F^{3}=F_{1}$ ? Регулярные слои можно связать гамильтоновым преобразованием прямого произведения $\mathbb{C P}_{x}^{2} \times \mathbb{C P}_{y}^{2}$. Это можно сделать следующим образом. Рассмотрим морсовскую функцию $g$ на $\mathbb{C P}_{w}^{2}$ такую, что ее гамильтоново векторное поле за конечное время сдвигает проективную прямую $l_{1}=\left\{w_{0}+w_{1}+w_{2}=0\right\}$ в проективную прямую $l_{0}=\left\{w_{1}+w_{2}\right\}$. Если зафиксировать морсовскую функцию $h_{1}$ на проективной прямой $l_{1}$, то она деформируется в морсовскую функцию $h_{0}$ на $l_{0}$. В то же время можно определить гамильтонову изотопию всего $\mathbb{C P}_{x}^{2} \times \mathbb{C P}_{y}^{2}$, порождаемую частичным подъемом $g$. Так как $\psi^{*} g$ не определена на базисном множестве $B \subset \mathbb{C P}_{x}^{2} \times \mathbb{C P}_{y}^{2}$ и не является гладкой на множестве особенностей Sing, возьмем две малые вложенные окрестности $B_{1} \supset B_{2}$ достаточно малых радиусов $1 \gg r_{1}>r_{2}>0$ вокруг

$$
\Delta\left(F_{1}, F_{2}\right)=B \cup \operatorname{Sing} \subset \mathbb{C P}_{x}^{2} \times \mathbb{C P}_{y}^{2}
$$

Тогда можно построить гладкую функцию $G$ на всем $\mathbb{C P}_{x}^{2} \times \mathbb{C P}_{y}^{2}$ по следующим правилам: $G=\psi^{*} g$ на $\mathbb{C P}_{x}^{2} \times \mathbb{C P}_{y}^{2}-B_{1}, G=0$ в $B_{2}$, функция $G$ гладко изменяется от $\partial B_{1}$ до $\partial B_{2}$. Нетрудно видеть, что гамильтоново действие $G$ на $\mathbb{C P}_{x}^{2} \times \mathbb{C P}_{y}^{2}$ сдвигает за

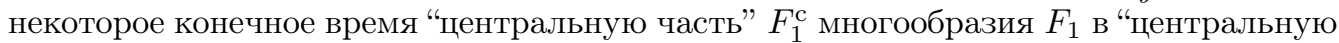
часть" $F_{0}^{\mathrm{c}}$ многообразия $F_{0}$, где под “центральной частью" мы понимаем

$$
F_{i}^{\mathrm{c}}=F_{i}-\left(F_{i} \cap B_{1}\right), \quad i=0,1 .
$$

Следовательно, имеет место следующее

ПрЕДЛОЖЕНИЕ 5. Каждый гладкий лагранжев тор в минимальном лагранжевом слоении на $F^{3}$, построенный в предыдущем разделе, гамильтоново изотопен некоторому гладкому лагранжеву тору из канонического лагранжева слоения торического вырождения $F_{0}$.

Для доказательства надо взять $r_{1}$ так, что данный тор не пересекает $B_{1}$; нетрудно видеть, что это возможно. Тогда гамильтонова изотопия, порождаемая $G$, сдвинет 
данный тор в некоторое подмногообразие в $F_{0}$, которое будет расслоено над соответствующей линией уровня функции $h_{0}$. По построению $G$ эта функция коммутирует с $F_{i}$ везде вне $B_{1}$, и это означает что полученный тор в $F_{0}$ лежит на совместном множестве уровней этих функций, а отсюда следует, что он будет лагранжевым в $F_{0}$. Остается заметить, что он порождается псевдоторической структурой по функции $h_{0}$, которая имеет в точности две критические точки, подлежащие в точности особым слоям отображения $\psi_{0}$, поэтому это слой канонического слоения.

Особый тор при переходе к пределу $t \rightarrow 0$ сжимается в изотропный тор в $F_{0}$, лежащий на компоненте граничного дивизора.

Последнее утверждение влечет следующий факт.

СлеДСТВИЕ. Когомологии Флоера $F H(S)$ гладкого слоя $S \subset F^{3}$ минимального лагранжева слоения совпадают с когомологиями Флоера некоторого слоя канонического лагранжева слоения многообразия $F_{0}$, которое является торическим върождением многообразия флагов $F^{3}$.

В самом деле, когомологии Флоера (вне зависимости от того, кто и как их определяет) должны быть стабильными относительно гамильтоновой изотопии, и все данные, используемые для вычислений $F H(S)$ на $F_{0}$ могут быть перенесены на $F^{3}$, что должно приводить к тому же самому ответу.

Интересно сравнить результаты данного раздела с результатами работы [6], так как именно эта интересная статья привлекла внимание автора настоящей работы к многообразию флагов.

\section{5. ЗАКЛЮЧЕНИЕ}

Конструкции, представленные в настоящей работе, приводят к еще одному важному вопросу лагранжевой геометрии комплексных многообразий флагов - к проблеме классификации монотонных лагранжевых торов с точностью до гамильтоновой изотопии. Эта проблема окончательно не решена даже для проективной плоскости $\mathbb{C P}^{2}$ (см. обсуждение в статье [3]), однако считается достоверным, что в этом случае существуют два типа монотонных лагранжевых торов, первый из которых доставляется торической структурой, в то время как второй, чекановского типа, может быть получен из псевдоторических рассмотрений [7].

В случае многообразия флагов $F^{3}$ имеется стандартный монотонный тор, представляемый слоем в слоении Гельфанда-Цейтлина. Однако рассмотрение псевдоторических торов приводит к другим монотонным торам, и естественно ожидать, что, как и в проективном случае, эти новые торы окажутся другими, "нестандартными".

Благодарности. Я хочу поблагодарить С. Белева, Д. Ору и Д. Орлова за полезные обсуждения и замечания. Особая благодарность Ю. Нохаре, чей доклад на конференции GEOQUANT-2009 (Luxembourg, September 7-11, 2009) дал толчок настоящей работе. Следовательно, я обязан поблагодарить и организаторов этой конференции, в первую очередь М. Шлихенмайера и А. Сергеева, что я делаю с огромным удовольствием. Работа выполнена при частичной финансовой поддержке Лаборатории алгебраической геометрии ГУ-ВШЭ (грант правительства РФ, дог. 11.G34.31.0023), РФФИ (гранты № 08-01-00095, 08-01-00392_а, 10-01-93113) и Программы поддержки ведущих научных школ (грант НШ-1987.2008.1). 


\section{Список литературы}

[1] M. Audin, Torus Actions on Symplectic Manifolds, Progr. Math., 93, Birkhäuser, Basel, 2004.

[2] С. А. Белев, Н. А. Тюрин, Матем. заметки, 87:1 (2010), 48-59.

[3] Н. А. Тюрин, ТМФ, 162:3 (2010), 307-333.

[4] V. Guillemin, S. Sternberg, J. Funct. Anal., 52:1 (1983), 106-128.

[5] D. Auroux, J. Gökova Geom. Topol., 1 (2007), 51-91, arXiv: 0706.3207.

[6] T. Nishinou, Y. Nohara, K. Ueda, Adv. Math., 224:2 (2010), 648-706, arXiv: 0810.3470.

[7] N. A. Tyurin, Twist tori and pseudotoric structures, arXiv: 1004.2574.

Поступила в редакцию 1.08.2010 